\title{
Corner Plus
}

National Cancer Institute

\section{Source}

National Cancer Institute. Corner Plus. NCI Thesaurus. Code C67053.

The average cell intensity for the sense probe cells used in the grid alignment process. 